\title{
Form und Funktion der doppelten Negation in deutschen Dialekten, mit einem Schwerpunkt im Oberdeutschen*
}

\author{
Ann-Marie Moser (Zürich)
}

\begin{abstract}
The article gives an overview of the form and function of negative concord in German dialects (Alemannic, Bavarian, Upper Franconian varieties, West Central German, East Central German, West Low German, East Low German, Silesian, East Pomeranian, Low Prussian), with a focus on Upper German. The study is based on spontaneous speech data from the 1950s until the 1980s and shows that negative doubling and negative spread (in German: 'doppelte Negation mit Satznegation' and 'doppelte Negation ohne Satznegation') are two different negation types, thus there is no correlation between them as generally assumed (Haspelmath 1997; Zeijlstra 2004). Furthermore negative doubling is not obligatory, but seems to be conditioned by pragmatics.
\end{abstract}

\section{$1 \quad$ Einleitung}

Bei der doppelten Negation (im Folgenden: dN), also dem Auftreten mehrerer Negationsmarker in einem Satz mit einfacher semantischer (und nicht sich gegenseitig aufhebender, positiver) Bedeutung, sind zwei verschiedene Typen möglich, die sich in Bezug auf die An- und Abwesenheit der Satznegation unterscheiden (cf. den Besten 1986: 205; van der Wouden/Zwarts 1993: 202). In den Beispielen (1) bis (3) tritt ein negatives Indefinitum (im Folgenden: n-Indefinitum) zusammen mit der verbalen Satznegation nicht auf. Diesen Typ bezeichne ich als , "dN mit Satznegation": 1

1. der macht dir die Arweit nie nich, er hat's schon Chahr und Taach versprochen der macht dir die arbeit nie NEG er hat-es schon jahr und tag versprochen ,Der macht dir nie die Arbeit, er hat es schon seit Jahren versprochen. (Obersächsisch; Threna, Lkr. Leipzig; Wörterbuch der obersächsischen Mundarten 1994: 298)

\footnotetext{
* Den HerausgeberInnen sowie den anonymen GutachterInnen danke ich für wertvolle Kommentare und Vorschläge, die den Beitrag deutlich verbessert haben. Im Folgenden werden Formen des grammatischen männlichen Geschlechts generisch verwendet.

Dieser Aufsatz stellt vorläufige Teilergebnisse meiner Dissertation vor.

${ }^{1}$ Der dN mit Satznegation entspricht engl. negative doubling, der dN ohne Satznegation negative spread.

Der Fettdruck in diesem und den folgenden Zitaten stammt, sofern nicht anders vermerkt, von AMM.
} 
2. et is keener nich da

es ist keiner NEG da

,Es ist keiner da.

(Brandenburgisch; Markee, Lkr. Havelland; Brandenburg-Berlinisches Wörterbuch 1985: 930)

3. ich sich khan mendsn nit

ich sehe keinen menschen NEG

,Ich sehe keinen Menschen.

(Ostfränkisch; Nürnberg; Gebhardt 1907: 306)

In den Beispielen (4) bis (6) liegen zwei n-Indefinita ohne Satznegation vor. Diesen Typ bezeichne ich als ,dN ohne Satznegation“:

4. Er het nie kei Geld

er hat nie kein geld.

,Er hat nie Geld.

(Niederalemannisch; Schutterwald, Lkr. Ortenaukeis; Braunstein 1978: 43)

5. däär hòd nia nex gesaid

der hat nie nichts gesagt

,Er hat nie etwas gesagt.

(Schwäbisch; Vogt 1977: 164)

6. Er tuet käm Mäntsche nü̈ü $z$ läid

er tut keinem menschen nichts zu leide

,Er tut keinem Menschen etwas zuleide.

(Hochalemannisch; Zürich; Weber 1987: 270)

Die dN mit Satznegation und die dN ohne Satznegation fallen unter den Oberbegriff der „doppelten Negation“. Zu den Negationsmarkern, die in einer dN stehen können, gehören die Satznegation sowie die nominalen n-Indefinita koa ,kein', niama ,niemand' und nicks ,nichts', das temporale Adverb nia ,nie“ und das lokale Adverb niama ,nirgends ‘. ${ }^{2}$ Sofern sich in einem Satz mehr als zwei Negationsmarker im Skopus der Negation befinden, wird die Form entweder zur dN mit Satznegation oder zur dN ohne Satznegation gerechnet. Dies hängt davon ab, ob die Satznegation vorhanden ist oder nicht. Beispiel (7) zählt so zur dN mit Satznegation, Beispiel (8) hingegen zur dN ohne Satznegation:

7. Wann koana koa Geid ned hod!

wenn keiner kein geld NEG hat

,Wenn keiner Geld hat!

(Mittelbairisch; Zehetner 1985: 149)

8. $s$ het no nie neamed nin(t) gseit

es hat noch nie niemand nichts gesagt

,Es hat noch nie jemand etwas gesagt.

(Bodenseealemannisch; Stahringen bei Radolfzell, Lkr. Konstanz; Staedele 1927: 53)

\footnotetext{
2 Die konkreten lautlichen Realisierungen unterscheiden sich natürlich von Dialekt zu Dialekt, die Distribution ist aber dieselbe.
} 
Im Folgenden soll auf Basis spontansprachlicher Daten auf die Verteilung der Formen der dN in den deutschen Dialekten sowie auf die Funktion der dN eingegangen werden. Entgegen bisheriger Annahmen (cf. Haspelmath 1997: 219; Zeijlstra 2004: 63) wird sich zeigen, dass die $\mathrm{dN}$ mit Satznegation und die dN ohne Satzegation zwei funktional verschiedene Negationsstrategien darstellen und voneinander unabhängig auftreten können. Desweiteren deuten meine Daten darauf hin, dass die dN mit Satznegation pragmatisch konditioniert ist.

Der Artikel ist wie folgt gegliedert: Nach einer Beschreibung von Korpus und Methode (Punkt zwei) werden die verschiedenen Formen der dN in den deutschen Dialekten vorgestellt (Punkt drei). Anschließend wird auf die Funktion der dN eingegangen, und zwar zunächst aus dem Blickwinkel der Semantik (4.1) und dann der Pragmatik (4.2). Der Aufsatz schließt mit einer Zusammenfassung (Punkt fünf).

\section{Korpus und Methode}

Es wurden drei verschiedene Korpora genutzt: das Zwirner-Korpus, das DDR-Korpus sowie das SSA-Korpus. Die meisten Sprecher verwenden den Basisdialekt, einige Wenige auch die regionale Umgangssprache. Im Rahmen der Untersuchung wurde nicht zwischen Basisdialekt und Regiolekt unterschieden.

Das Zwirner-Korpus besteht aus digitalisierten und transkribierten Interviews, welche über die Datenbank für Gesprochenes Deutsch des Instituts für deutsche Sprache (IDS Mannheim) zur Verfügung stehen. Das Korpus umfasst Interviews in größtenteils monologischer Form, die unter der Leitung von Eberhard Zwirner in den alten Bundesländern durchgeführt wurden (Zeitraum: 1955 bis 1972). Ziel war, die im Alltag gebrauchte Sprache des Sprechers, also die Sprache bei sich zu Hause oder an seinem Arbeitsplatz, aufzunehmen (cf. Zwirner/Bethge 1958: 19). Für diesen Artikel wurden alle verfügbaren transkribierten Interviews nach der dN durchsucht, insgesamt ca. 2.440 Interviews mit autochthonen Sprecher unterschiedlicher Altersgruppen (cf. IDS Mannheim: Datenbank für Gesprochenes Deutsch: Zwirner-Korpus). Darunter fallen auch Aufnahmen mit ehemals Vertriebenen, also Sprechern, die kriegsbedingt ihre Heimat verlassen mussten und eine Mundart wie Schlesisch, Ostpommersch oder Niederpreußisch beherrschen. Da bei diesen Sprechern „ein unverkennbares Bemühen hervor[trat], das Heimatidiom zu unterdrücken, um auch auf sprachlicher Ebene leichteren Anschluß an die neue Gemeinschaft zu finden“ (Bellmann 1964: 70f.) und sie „seit ca. zwei Jahrzehnten um rasche soziale Integration bemüht [waren], wobei das Vermeiden der stark stigmatisierten Merkmale der Herkunftsvarietäten eine herausragende Rolle spielte“ (Schmidt/Herrgen 2011: 122), wurden bei den Sprechern von Vertriebenenmundarten nur jene ab 60 Jahre aufgenommen (cf. Bellmann 1964: 70). Hintergrund hierfür war die Beobachtung, dass jüngere Sprecher, die nach der Vertreibung noch längere Zeit berufstätig waren, sich sprachlich sehr stark akkomodiert hatten (cf. Bellmann 1964: 70).

Um auch die ostdeutschen Dialektgebiete in die Untersuchung einzubeziehen, wurde neben dem Zwirner-Korpus ein weiteres Korpus hinzugezogen, das das ostdeutsche Pendant zum Zwirner-Korpus darstellt. Das DDR-Korpus umfasst „Proben der Dialekte Ostdeutschlands“ und wurde ,[i]n Anlehnung an die Aufnahmeaktion des damaligen Deutschen Spracharchivs“ (cf. IDS Mannheim: Datenbank für Gesprochenes Deutsch: DDR-Korpus) durchgeführt. Hier liegen also, wie im Zwirner-Korpus, vorwiegend monologische Aufnahmen mit autochthonen

ISSN 1615-3014 
Sprechern aus unterschiedlichen Altersgruppen vor. Die Aufnahmen umfassen einen Zeitraum von 1960 bis 1968 und entstanden damit zur selben Zeit wie die Aufnahmen für das ZwirnerKorpus. Aus dem DDR-Korpus stammen 117 transkribierte Aufnahmen.

Da der Schwerpunkt des Artikels auf den Sprechern mit dN aus dem oberdeutschen Sprachgebiet liegt, wurde noch ein drittes Korpus hinzugezogen, da die Anzahl der alemannischen Sprecher aus dem Zwirner-Korpus, im Vergleich zu den bairischen Sprechern, sonst sehr niedrig gewesen wäre. Diese Interviews stammen aus dem Südwestdeutschen Sprachatlas (SSA) und wurden in den 1970er und 1980er Jahren mit Dialektsprechern aus dem Gebiet der ehemaligen Regierungsbezirke Südbaden und Südwürttemberg durchgeführt (cf. König/Schrambke 1999: 103f.). ${ }^{3}$ Im Gegensatz zum Zwirner-Korpus liegen im Fall des SSAs mehrheitlich Gruppeninterviews vor, sodass der Gesprächsanteil mancher Sprecher teilweise nur aus wenigen Gesprächseinsätzen (z. B. nur drei Gesprächseinsätze von geringem Umfang bei einem Sprecher) besteht. Diese Sprecher wurden nicht in die Untersuchung aufgenommen (weder als Sprecher mit dN noch als Sprecher ohne dN). Es wurden nur jene Sprecher ausgewählt, die über 60 oder über mehr als 60 Gesprächseinsätze verfügen. Im selteneren Fall einer eher monologischen Gesprächssituation mit nur einem, maximal zwei Informanten wurden jedoch auch weniger als 60 Gesprächseinsätze pro Informant akzeptiert, sofern der Gesprächsanteil entsprechend länger war. In methodologischer Hinsicht mag dem aufmerksamen Leser bereits aufgefallen sein, dass von einzelnen Sprechern die Rede ist: Dies ist wichtig, denn ich habe meine Daten nicht nach der Häufigkeit der Form „dN“ ausgewertet ohne Berücksichtigung des einzelnen Sprechers (also in etwa: So häufig tritt die dN mit Satznegation auf, so häufig die $\mathrm{dN}$ ohne Satznegation). Es ist also in diesem Sinn keine korpuslinguistische Untersuchung. Stattdessen interessiert mich, ob (a) ein Sprecher die dN verwendet oder nicht, ob (b) er beide Formen verwendet oder nur eine von beiden, und ob (c) er diese Form(en) frei optional oder konditioniert verwendet. Es geht also um die Kompetenz, nicht die Performanz des Sprechers: Ich betrachte die Grammatik jedes einzelnen Sprechers mit dN; unter „Grammatik“ verstehe ich "a set of construction types used by an individual” (Bresnan/Deo/Sharma 2007: 304). Erst durch die Untersuchung der interlektalen Variation zwischen Sprechern und der idiolektalen Variation innerhalb eines Sprechers zeigt sich nämlich, dass die $\mathrm{dN}$ mit Satznegation und die $\mathrm{dN}$ ohne Satznegation funktional verschieden sind und nicht, wie bisher in der typologischen Forschung angenommen (cf. Haspelmath 1997; Zeijlstra 2004), in einem Abhängigkeitsverhältnis zueinander stehen.

\section{Zur Form und Verbreitung der dN}

Für die Sprecher aller Varietäten gilt: (1) Die dN ohne Satznegation ist bis auf sehr wenige Ausnahmen nur in adjazenter Stellung belegt. (2) Die dN mit Satznegation ist dagegen sowohl adjazent als auch nicht-adjazent möglich: Im Fall der nicht-adjazenten dN befindet sich das nIndefinitum im Vorfeld, die Satznegation am rechten Rand des Mittelfeldes; im Fall der adjazenten $d N$ befinden sich Satznegation und n-Indefinitum gemeinsam im Mittelfeld. Die Satznegation folgt dem n-Indefinitum; bis auf wenige Ausnahmen wird die dN mit Satznegation

\footnotetext{
3 Ich danke Peter Auer für den Zugang zu den Interviews des SSA.
} 
in allen Varietäten ausschließlich mit dem n-Indefinitum koa ,kein` bzw. keen ,kein` etc. realisiert.

Vergleiche so folgende Beispiele: ${ }^{4}$

9. dort kann man nirgends keinen Grund herbringen.

,Dort kann man nirgends Grund hinbringen.

(Alemannisch; Horben, Lkr. Breisgau- Hochschwarzwald; ZW: E_00042_S_00049)

10. Ja, eben. Ich bin halt kein Weintrinker nicht, ich mag bloß ein Bier.

,Ja, eben. Ich bin kein Weintrinker, ich mag nur Bier.'

(Bairisch; Weltenburg, Lkr. Kelheim; ZW: E_01454_S_01586)

\section{Kein Wein tat man da nicht kennen!}

,Keinen Wein kannte man da!

(Westmitteldeutsch; Hirzenhain, Lkr. Wetteraukreis; ZW: E_03242_S_03386)

12. kein neues konnten wir uns nicht kaufen

,Kein Neues konnten wir uns kaufen.

(Ostmitteldeutsch; Reisdorf, Lkr. Weimarer Land; DR: E_00432_SE_0001)

13. Ich bin vierundneunzig abgegangen, da gab es noch hier keine Fahrräder nicht.

,Ich bin vierundneunzig abgegangen, da gab es hier noch keine Fahrräder.'

(Westniederdeutsch; Ascheberg, Lkr. Coesfeld; ZW: E_02977_S_03121)

14. da gab es ja keine festen Straßen nicht.

„Da gab es keine festen Straßen.

(Ostniederdeutsch; Eslohe, Lkr. Hochsauerlandkreis; ZW: E_03038_S_03182)

15. Ja, kein Erntefest haben wir ja nicht gefeiert dort, gelt.

,Ja, kein Erntefest haben wir dort gefeiert.'

(Schlesisch; Liebau (Lubawka) in Niederschlesien, heuiges Polen; ZW:

E_02840_S_02982)

16. Hausfrau hat viel zu tun, das weiß keiner nicht.

,Eine Hausfrau hat viel zu tun, das weiß keiner.

(Niederpreußisch; Peyszeln/Lwowskoje in Kaliningrad, heutige Russische Föderation; ZW: E_04557_S_04704)

17. Als der Russe kam wurde von der Einsegung nichts nicht, nicht

,Als der Russe kam, wurde aus der Einsegnung nichts. ‘

(Ostpommersch; Henkenhagen (Ustronie Morskie) in Westpommern, heutiges Polen; ZW: E_04732_S_04879)

Was die Verbreitung der dN betrifft, so gibt Tabelle 1 einen Überblick über die Anzahl der Sprecher mit dN in Relation zur Gesamtzahl untersuchter Sprecher. Die Angaben in Prozent wurden in diesem Fall sowie für alle weiteren Angaben in Prozent auf eine Stelle nach dem Komma aufgerundet. Die einzelnen Dialektverbände umfassen dabei die folgenden Varietäten: Alemannisch (inkl. Elsass) mit Nieder- und Hochalemannisch sowie Schwäbisch, Bairisch mit Nord-, Mittel- und Südbairisch, die oberfränkischen Varietäten mit Süd- und Ostfränkisch, Westmitteldeutsch mit Mittel- und Rheinfränkisch, Ostmitteldeutsch mit Obersächsisch und

\footnotetext{
${ }^{4}$ In den Beispielen aus dem Zwirner-Korpus wird die standarddeutsche Transkription übernommen, da für diesen Artikel die Syntax (und nicht etwa die Morphologie) relevant ist.
} 
Thüringisch, Westniederdeutsch mit West- und Ostfälisch sowie Nordniederdeutsch und schließlich Ostniederdeutsch mit Mecklenburgisch-Vorpommersch und Brandenburgisch. Die Vertriebenenmundarten, i. e. Schlesisch, Niederpreußisch und Ostpommersch, werden gesondert und nicht zum Ostmittel- bzw. Ostniederdeutschen gezählt, da sie sich hinsichtlich der Sprecherzahlen teilweise deutlich vom Ostmitteldeutschen (bzgl. Schlesisch) und Ostniederdeutschen (bzgl. Niederpreußisch und Ostpommersch) unterscheiden. ${ }^{5}$

\begin{tabular}{lcc}
\hline & Sprecher mit dN & gesamt untersuchte Sprecher \\
\hline Alemannisch & & 453 \\
Bairisch & $14(18 \%)$ & 295 \\
oberfränkische Varietäten & $47(22 \%)$ & 209 \\
Westmitteldeutsch & $22(6 \%)$ & 400 \\
Ostmitteldeutsch & $11(10 \%)$ & 114 \\
Westniederdeutsch & $28(3 \%)$ & 1054 \\
Ostniederdeutsch & $5(7 \%)$ & 71 \\
\hline Schlesisch & $13(22 \%)$ & 58 \\
Niederpreußisch & $21(15 \%)$ & 142 \\
Ostpommersch & $4(4 \%)$ & 102 \\
\hline
\end{tabular}

Tabelle 1: Sprecher mit und ohne dN (Überblick)

Es ist deutlich zu erkennen, dass die $\mathrm{dN}$ bei Sprechern aller Varietäten (noch) möglich ist; gleichzeitig liegt aber eine deutliche Nord-Süd-Asymmetrie vor: Die meisten Sprecher mit dN (ohne die Vertriebenenmundarten) finden sich im Oberdeutschen und den oberfränkischen Varietäten, beide niederdeutschen Varietäten weisen weniger Sprecher mit dN auf als das Mitteldeutsche. Das erstaunt nur wenig, da die oberdeutschen Mundarten prestigeträchtiger sind als jene im Norden: „In der oberdeutschen Dialektregion genießt mundartliches Sprechen eine recht hohe Wertschätzung [...]. Im niederdeutschen Dialektraum haben Mundarten einen relativ niedrigen Status [...]. Die mitteldeutsche Dialektregion nimmt auch in dieser Hinsicht eine Zwischenstellung ein.“ (Barbour/Stevenson 1998: 88). Neben der Nord-Süd-Asymmetrie ist auch eine leichte Ost-West-Asymmetrie zu beobachten: Es sind mehr Sprecher einer östlichen Mundart mit dN belegt (in Relation zur Gesamtzahl untersuchter Sprecher) als Sprecher einer westlichen Mundart. Auf den ersten Blick könnte man annehmen, dass hier möglicherweise die Nähe zu den slawischen Sprachen bzw. speziell zum Russischen (als dNSprache) für die Varietäten auf dem Gebiet der ehemaligen DDR eine Rolle spielen mag. Was den Einfluss des Russischen anbelangt, so nehmen jedoch Barbour/Stevenson (1998: 189) an, dass die Divergenzen zwischen Ost und West v. a. die Lexik und nicht die grammatische Struktur betreffen (cf. Barbour/Stevenson 1998: 195). Im Schlesischen liegen schließlich mit

\footnotetext{
${ }^{5}$ Die Dialekteinteilung erfolgte auf Basis der Mundart des Sprechers, die im Korpus bereits angegeben war.

${ }^{6}$ Aufteilung nach Korpus: Im SSA-Korpus insgesamt 317 Sprecher, davon 60 mit dN (19\%); im Zwirner-Korpus insgesamt 196 Sprecher, davon 27 mit dN (14\%).
} 
13 Sprechern (22\%) und im Niederpreußischen mit 21 Sprechern (15\%) vergleichsweise hohe Zahlen vor, im Ostpommerschen mit vier Sprechern (4\%) dagegen nur sehr niedrige. Im Schlesischen wurde die $\mathrm{dN}$ somit noch relativ häufig von den Sprechern verwendet, im Ostpommerschen kaum. Angsichts dieser Zahlen liegt es nahe anzunehmen, dass die dN ein rein basisdialektales Merkmal ist (und im Regiolekt nicht möglich ist).

Folgende Abbildung zeigt die Mundartorte der Sprecher mit dN; die Sprecher der Vertriebenenmundarten sind nicht abgebildet.

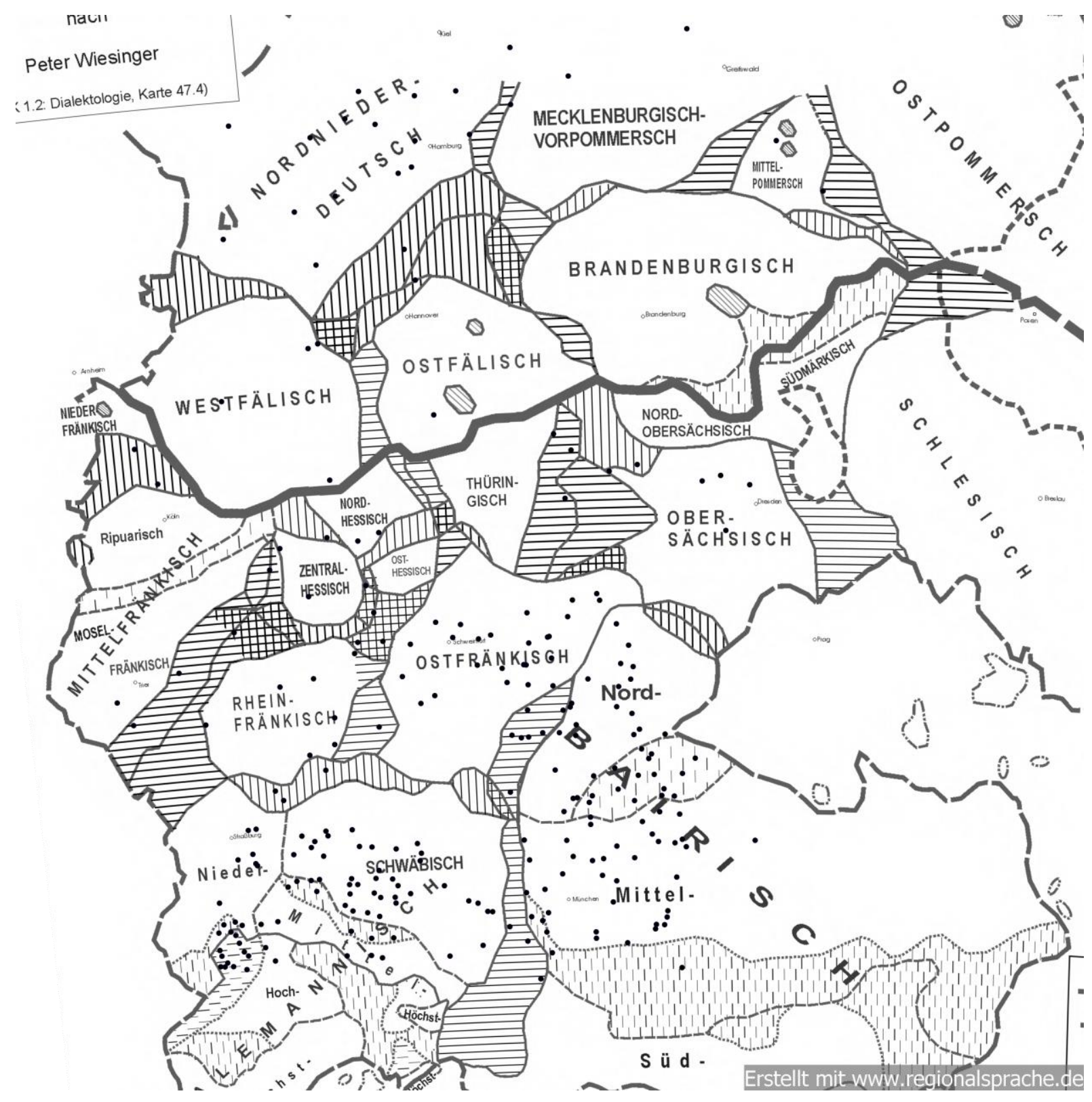

Abbildung 1: Die Verteilung der Mundartorte der Sprecher mit dN

Tabelle zwei konzentriert sich nun ausschließlich auf die Sprecher mit dN, sie stellt also eine Art Zoom dar. Die Sprecher sind aufgeteilt nach: (a) Sprecher mit dN mit Satznegation, (b) Sprecher mit dN ohne Satznegation, (c) Sprecher mit beiden Typen in unterschiedlichen Sätzen. 


\begin{tabular}{lccc}
\hline Sprecher mit: & dN mit Satznegation & dN ohne Satznegation & beide Typen \\
\hline Alemannisch ${ }^{7}$ & $13(16 \%)$ & $60(74 \%)$ & $8(10 \%)$ \\
Bairisch & $126(89 \%)$ & $3(2 \%)$ & $13(9 \%)$ \\
Oberfränkische Varietäten & $39(83 \%)$ & $5(11 \%)$ & $3(6 \%)$ \\
Westmitteldeutsch & $13(59 \%)$ & $8(36 \%)$ & $1(5 \%)$ \\
Ostmitteldeutsch & $11(100 \%)$ & - & - \\
Westniederdeutsch & $27(96 \%)$ & $1(4 \%)$ & - \\
Ostniederdeutsch & $5(100 \%)$ & - & $1(8 \%)$ \\
\hline Schlesisch & $12(92 \%)$ & - & $1(5 \%)$ \\
Niederpreußisch & $17(81 \%)$ & $3(14 \%)$ & - \\
Ostpommersch & $4(100 \%)$ & - & - \\
\hline
\end{tabular}

Tabelle 2: Sprecher mit dN (Einteilung nach Negationsform) ${ }^{8}$

Wir sehen, dass die dN mit Satznegation in allen Varietäten belegt ist, und zwar in allen Varietäten bis auf das Alemannische als bevorzugte Negationsstrategie. Die dN ohne Satznegation ist dagegen nicht in allen Varietäten anzutreffen und nur im Alemannischen bei der Mehrheit der Sprecher die präferierte Form. In keiner Varietät treten Sprecher, die beide Negationsstrategien verwenden, mehrheitlich auf, und nur bei der Hälfte der Sprecher ist dieser Typ überhaupt belegt.

Im oberdeutschen Sprachraum finden wir also mit dem Alemannischen eine Varietät, die die $\mathrm{dN}$ vor allem in Form der $\mathrm{dN}$ ohne Satznegation realisiert, und mit dem Bairischen eine Varietät, die die $\mathrm{dN}$ fast ausschließlich in Form der $\mathrm{dN}$ mit Satznegation realisiert. Dabei nutzen die Sprecher nicht den jeweils anderen Typ, auch wenn die Möglichkeit dazu besteht (systematisch überprüft wurden die alemannischen und bairischen Sprecher), wie die folgenden Beispiele zeigen:

18. ein Sprecher des Bairischen:

a. Weil ich habe da keine Angst nicht, daß ich mir woanders auch einarbeite und woanders verdiene, nicht.

b. Was ich heute noch bin, das geht niemand etwas an

(Scharmassing, Kr. Regensburg; ZW: E_01455_S_01587)

\footnotetext{
${ }^{7}$ Die genaue Aufteilung nach Korpus: Im Zwirner-Korpus verwenden fünf Sprecher die dN mit Satznegation, elf Specher die dN ohne Satznegation und fünf Sprecher beide Typen. Im SSA-Korpus liegen acht Sprecher mit dN mit Satznegation vor, 49 Sprecher mit dN ohne Satznegation und drei Sprecher mit beiden Typen.

${ }^{8}$ Wie bereits erwähnt (Punkt zwei), geht es nicht um die Anzahl der Belege für die dN bei einem Sprecher (reine Performanz), sondern es sollen aufgrund der Performanz Rückschlüsse auf die Kompetenz gezogen werden. Es werden also (a) Sprecher mit mehr als 60 Gesprächseinsätze berücksichtigt und (b) Sprecher berücksichtigt, bei denen die dN nur einmal belegt ist. Dies ist bei der dN mit Satznegation nur selten der Fall, bei der dN ohne Satznegation hingegen nicht ungewöhnlich, da zwei Indefinita in einem Satz einfach nicht so häufig auftreten wie ein n-Indefinitum alleine.
} 
19. ein Sprecher des Bairischen:
a. kein junges Frauenzimmer will überhaupt nicht mehr hinein in den Stall
b. Nein, hat die Resi nie Zeit gehabt

(Oberhummel, Lkr. Freising; ZW: E_01488_S_01620)

20. ein Sprecher des Alemannischen:
a. Wenn du nur mal nie keine Flinte gehabt hättest, hat sie als gesagt damals.
b. Ja, bei uns haben sie kein Holz gemacht, die Franzosen.
(Gamshurst-Achern, Lkr. Ortenaukreis; ZW: E_00548_S_00627)

21. ein Sprecher des Alemannischen:
a. von einem Schmied da kommt nie nichts in einer Illustrierten
b. das das gibt doch kein schmied das gibt ein schneider hat sie gesagt (Waldmössingen, Lkr. Rottweil; SSA)

Eine ähnliche gemischte Situation findet sich übrigens in früheren Sprachstufen des Niederdeutschen: Im Altniederdeutschen ist so die $\mathrm{dN}$ mit Satznegation belegt, im Mittelniederdeutschen hingegen die dN ohne Satznegation (cf. Breitbarth 2013: 217). Die dN mit Satznegation und die dN ohne Satznegation stellen somit im Oberdeutschen zwei struktuell verschiedene Negationstypen dar, i. e. es besteht keine Korrelation zwischen beiden Typen, wie man in der typologischen Forschung annimmt: ,(a) If in a language negative pronouns do not co-occur with verbal negation, they also do not co-occur with each other, and conversely, (b) if negative pronouns do not co-occur with each other, they do not co-occur with verbal negation either.“ (Haspelmath 1997: 219; ähnlich auch bei Zeijlstra 2004: 63)

Was den Status von nimmer, nicht-mehr' in dN betrifft, so finden wir hier Sprecher vor, bei denen das Lexem aufgrund seiner Stellung im Satz als Satznegation zu rechnen ist; gleichzeitig fungiert es auch bei manchen Sprechern als Adverbial und stellt hier also ein n-Indefinitum dar. In den folgenden Beispielen (22) und (23) zähle ich nimmer zur Satznegation, da es bei den Sprechern nicht gemeinsam mit der Satznegation belegt ist sowie dem n-Indefinitum folgt (cf. ähnlich auch Weiß 1998: 206f.)

22. Die Roß also keine schlechten heute sieht man jetzt nimmer, sondern äh man sieht jetzt die besseren Roß

,Keine schlechten Pferde sieht man heute mehr, man sieht jetzt bessere Pferde“

(Alemannisch; Buchloe, Lkr. Ostallgäu; ZW: E_00883_S_01007)

23. ich habe meist überhaupt keine Sense nicht mehr gehabt

"Ich habe meistens keine Sense mehr gehabt.'

(Westniederdeutsch; Geestland, Lkr. Cuxhaven; ZW: E_04574_S04721)

In den Beispielen (24) und (25) stellt nimmer aufgrund seiner Stellung im Satz ein nIndefinitum dar: Es tritt mit Satznegation bzw. mit einem anderen n-Indefinitum auf und fungiert in beiden Fällen als adverbiales n-Indefinitum.

24. Man kann sich nimmer nicht darauf verlassen.

,Man kann sich nicht mehr darauf verlassen.

(Westniederdeutsch; Niesgrau, Lkr. Schleswig-Flensburg; ZW: E_02414_S02550) 
25. und dann war der Hopfen sozusagen ein Judenhandel, sonst hat er nimmer keine gekauft ,Und dann war der Hopfen sozusagen ein Judenhandel, sonst hat ihn keiner mehr gekauft (Alemannisch; Markdorf-Ittendorf, Lkr. Bodenseekreis; ZW: E_00103_S_00127)

\section{$4 \quad$ Zur Funktion der dN mit Satznegation: optional oder konditioniert?}

\subsection{Ein Erklärungsansatz aus der Semantik?}

Erklärungen in Bezug auf die Funktion der dN mögen zwar im genauen Wortlaut variieren, allerdings ändert sich der Grundgedanke, der im Sprecher-Hörer-Verhältnis und dem Verhältnis von Explizitheit und Ökonomie einer Äußerung angesiedelt ist, nicht. Schon bei Jespersen ist zu finden: "[I]t requires greater mental energy to content oneself with one negative, which has to be remembered during the whole length of the utterance both by the speaker and by the hearer, than to repeat the negative idea (and have it repeated) whenever an occasion offers itself." (Jespersen 1917: 71) Ähnlich auch bei Bernini/Ramat (1996: 188), die die Funktion der $\mathrm{dN}$ in ihrer größeren Explizitheit gegenüber der einfachen Negation sehen. Nach Abraham (2000: 223f.) erfüllt die dN die Aufgabe, dass der Sprecher sicher sein kann, dass dem Hörer die wichtigste rhematische Information, nämlich die Negation, nicht entgeht. Aus diesem Grund wird sie verdoppelt. Und aus generative Perspektive wird dem Sprecher durch die dN auf der Oberflächenstruktur angezeigt, welche Konstituenten sich im Skopus der Negation befinden. Sprecher von Sprachen ohne dN müssen dies hingegen inferieren (cf. Bayer 1990: 23).

Diese Erklärungen können aber nicht die Optionalität der dN mit Satznegation erklären: Die adjzente dN mit Satznegation wird von den Sprechern nicht in allen möglichen Kontexten verwendet, cf. so z. B. die beiden Sprecher des Bairischen:

26. a. den Jörg NAME, der hat bis heute noch keine Zentrifuge nicht

b. da gibt's keinen gescheiten Topfen, nicht wahr

(Bichl, Lkr. Bad Tölz-Wolfratshausen; ZW: E_00928_S_01052)

27. a. Der Bauer kriegt keine Arbeiter nicht mehr, weil-allesamt gehen sie in die Stadt.

b. Dann brauchen sie keine Kollektivwirtschaft mehr herführen, die kriegen wir am [auf dem] schönsten Weg, nicht.

(Regenstauf, Lkr. Regensburg; ZW: E_01468_S_01600)

Da die dN mit Satznegation nur optional verwendet wird, stellt sich die Frage, ob freie Optionalität vorliegt oder die Verwendung der $\mathrm{dN}$ in einer bestimmten Form restringiert ist. Weiß (1998: 200f.) nimmt an, dass die $\mathrm{dN}$ nicht rein optional, i. e. in freier Variation, auftritt. Seine (semantische) Erklärung ist im Rahmen der „Mapping Hypothese“ der Discourse Representation Theory nach Diesing (1992), Kamp (1981) und Heim (1982) angesiedelt: Ihrzufolge kann zwischen starken quantifizierenden und schwachen nicht-quantifizierenden Indefinita (und damit Lesarten) unterschieden werden; je nach syntaktischer Position liegt die eine oder die andere Lesart vor. Die beiden Sätze (28) und (29) geben ein Beispiel mit und ohne $\mathrm{dN}$, in (30a) und (30b) liegen die beiden verschiedene Lesarten vor (Beispiele nach Weiß 1998: 214f.). Für (28) ist nur Lesart (30a) möglich, da das n-Indefinitum ein schwacher Quantor ist und sich im Skopus von modalem und negativem Operator befindet. Das n-Indefinitum hat also kein quantifikatorisches Potential. In (29) sind hingegen beide Lesarten (30a) und (30b) 
möglich. Lesart (30a) ist möglich, wenn man tiefenstrukturell ein ned ,nicht" annimmt, das aber in der phonetischen Form (PF) getilgt wurde. Lesart (30b) ist möglich, wenn koa Beispiel ,kein

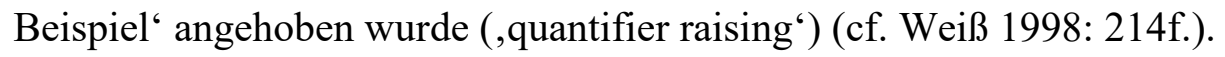

28. wai koa Beispiel ned bekannt sa muaß.

,weil kein Beispiel bekannt sein muss.

29. wai koa Beispiel bekannt sa muaß.

,weil kein Beispiel bekannt sein muss.

30. a. Es ist nicht notwendig, dass ein Beispiel bekannt ist.

b. Es gibt kein Beispiel, das bekannt sein muss.

Die Funktion der dN besteht also darin, anzuzeigen, dass nur eine Lesart möglich ist und dass das n-Indefinitum keinen Quantor darstellt. Das bedeutet, dass nur die existentielle Lesart möglich ist und keine universelle Interpretation des n-Indefinitums. Die beiden verschiedenen Lesarten sollen nun am Beispiel von zwei Target-Sätzen aus einer Fragebogenerhebung anschaulich erklärt werden. Zur Überprüfung der Hypothese wurde hierfür eine Fragebogenumfrage in Wall (Lkr. Miesbach, Oberbayern) mit 18 Informanten, Sprechern des Basisdialekts, durchgeführt. ${ }^{9}$ Die Target-Sätze und der Kontext wurden von Weiß (1998: 214221) übernommen (die Target-Sätze wörtlich, beim Kontext die Idee).

Die erste Aufgabe (Nr. 18) lautet wie folgt:

18. Oma Hilde überlegt, was sie ihrem Enkelkind Hans zu Weihnachten schenken soll. Sie möchte ihm eigentlich wieder einen Pullover stricken, das hat sie seit seiner Geburt immer so an Weihnachten gemacht. Die Mutter hat ihr aber gebeichtet, dass der Hans noch nie einen selbstgestrickten Pullover angezogen hat (die Wolle kratzt zu sehr). Die Oma sagt zum Opa, dass sie ihm dieses Jahr etwas anderes schenken muss, ...

Bitte geben Sie für jeden der folgenden Sätze an, wie natürlich er für Sie klingt:

\begin{tabular}{|ll|l|l|l|}
\cline { 3 - 5 } \multicolumn{1}{c|}{} & $\begin{array}{l}\text { sehr } \\
\text { natürlich }\end{array}$ & möglich & $\begin{array}{l}\text { völlig } \\
\text { unmöglich }\end{array}$ \\
\hline 1) & ... wai da Hans koan Pullover oziang mog. & $\square$ & $\square$ & $\square$ \\
\hline 2$)$ & ... wai da Hans koan Pullover ned oziang mog. & $\square$ & $\square$ & $\square$ \\
\hline
\end{tabular}

Welche Variante ist für Sie die natürlichste? Nr.

Würden Sie den Satz normalerweise in einer Form sagen, die nicht aufgeführt ist? $\square$ ja $\square$ nein

Wenn "ja": Bitte notieren Sie hier den Satz so, wie Sie ihn normalerweise sagen würden:

Die „,korrekte“ Antwort mit der richtigen Lesart für diese Aufgabe ist nach Weiß (1998: 217) der Target-Satz 1) ohne Satznegation. So liegt hier nämlich die Präsupposition vor, dass es

\footnotetext{
${ }^{9}$ Die Umfrage selbst wurde von den Studierenden des Hauptseminars «Dialektsyntax» (Leitung: Guido Seiler und Thilo Weber) durchgeführt. Ich danke den Seminarleitern und den Studierenden für die Aufnahme meiner Fragen in den Fragebogen.
} 
bestimmte Pullover gibt, Hans sie aber generell nicht anziehen möchte; i. e. dass es keinen Pullover gibt, den er anziehen möchte. Target-Satz 2) mit Satznegation wäre falsch, da sich diese Lesart nur auf einen bestimmten Pullover bezieht. Das ist hier jedoch nicht der Fall, denn Hans mag generell nicht die gestrickten Pullover der Oma.

Nun zur zweiten Aufgabe (Nr. 23):

23. Ludwig muss bei runden Geburtstagen in seiner Familie normalerweise immer die Festrede halten, und die Vorbereitung dafür nimmt jedes Mal viel Zeit in Anspruch. Dieses Mal übernimmt das aber zum Glück sein Bruder. Ludwig freut sich darüber und sagt zu seiner Frau:

Bitte geben Sie für jeden der folgenden Sätze an, wie natürlich er für Sie klingt:

\begin{tabular}{|l|l|l|l|l|}
\cline { 2 - 4 } \multicolumn{1}{c|}{} & $\begin{array}{l}\text { sehr } \\
\text { natürlich }\end{array}$ & möglich & $\begin{array}{l}\text { völlig } \\
\text { unmöglich }\end{array}$ \\
\hline 1) & $\begin{array}{l}\text { Guad, dass'e koa Redn } \\
\text { hoidn brauch. }\end{array}$ & $\square$ & $\square$ & $\square$ \\
\hline $\begin{array}{l}\text { Guad, dass'e koa Redn } \\
\text { ned hoidn brauch. }\end{array}$ & $\square$ & $\square$ & $\square$ \\
\hline
\end{tabular}

Welche Variante ist für Sie die natürlichste? Nr.

Würden Sie den Satz normalerweise in einer Form sagen, die nicht aufgeführt ist?

$\square$ ja $\square_{\text {nein }}$

Wenn "ja": Bitte notieren Sie hier den Satz so, wie Sie ihn normalerweise sagen würden:

In dieser Aufgabe sollte nach Weiß (1998: 216) der Target-Satz 2) mit Satznegation angekreuzt werden: Er drückt aus, dass sich jemand darüber freut, dass er irgendeine Rede nicht halten muss; i. e. Ludwig hält normalerweise Reden, aber diesmal eben nicht. Target-Satz 1) präsupponiert dagegen (generell) die Existenz von Reden, die nicht gehalten werden müssen.

Tabelle 3 zeigt die Antworten der Informanten, wobei die nach Weiß zu erwartende Antwort pro Aufgabe mit Fettdruck markiert ist: Aufgabe 23, in welcher nur die Version mit dN möglich sein sollte, haben fünf von 18 Informanten als ,,natürlich“ "bewertet. Bei Aufgabe 18, in welcher die Version ohne dN richtig ist, haben drei der Informanten die $\mathrm{dN}$ als „natürlich“ bewertet. Diese Ergebnisse sprechen nicht für Weiß` Erklärung (auch wenn hier natürlich die Kompetenz und nicht die Performanz der Sprecher getestet wurde und das Ergebnis nicht repräsentativ ist). Im Folgenden soll daher eine andere Erklärung, diesmal aus der Pragmatik, vorgeschlagen werden.

\begin{tabular}{llccc}
\hline & & natürlich & möglich & unmöglich \\
\hline Aufgabe 18 & koan Pullover & 18 & - & - \\
& koan Pullover ned & 3 & 12 & 3 \\
\multirow{2}{*}{ Aufgabe 23 } & koa Redn & 14 & 4 & - \\
& koa Redn ned & 5 & 12 & 1 \\
\hline
\end{tabular}

Tabelle 3: Ergebnis der Fragebogenerhebung 


\subsection{Ein pragmatischer Erklärungsansatz}

Im Englischen (und Katalanischen, Russischen und Japanischen) spielen bei der Wahl des Indefinitums die Sprechererwartungen eine Rolle (cf. Haspelmath 1997: 82-86). In (31) erwartet oder hofft der Sprecher, dass die Antwort auf seine Frage positiv ausfallen wird, sodass some verwendet wird. In (32) erwartet der Sprecher, dass die Antwort negativ ausfallen wird, sodass nur any möglich ist (cf. Haspelmath 1997: 82). Die Beispiele in (31) und (32) selbst stammen von Lakoff (1969: 609f.), werden aber hier aufgrund der besseren Verständlichkeit in der leicht veränderten Form von Haspelmath (1997: 82f.) übernommen. ${ }^{10}$

31. Do you think those men want to do some work? (Because my road needs to be repaired.)

32. Do you think those men want to do any work? (Because they've been standing around all morning telling dirty jokes.)

Auch bei Konditionalen spielen Sprechererwartungen eine Rolle: Wenn der Sprecher erwartet oder annimmt, dass die Bedingung erfüllt wird, i. e. wenn die Präsupposition wahr ist, wird some verwendet (33); wenn dagegen erwartet wird, dass sich die Annahme nicht bestätigt, wird any verwendet (34) (cf. Haspelmath 1997: 83):

33. Unicorns are mythical beasts: if John sees any <?? some > unicorns out there, I'll eat my hat.

34. If John sees some <?? any > goldfish in that tank, it's not surprising: there are lots of them in there.

Lässt sich diese Idee - dass die dN nur vor einem bestimmten Erwartungshorizont geäußert wird - auch auf die dN mit Satznegation übertragen?

Es hat den Anschein, als werde die $\mathrm{dN}$ im Bairischen nur vor einem positiven Erwartungshorizont geäußert. ${ }^{11}$ Betrachten wir also folgenden Interviewausschnitt (35) vor diesem Hintergrund: In 0010 bestätigt S2 den Kommentar von S1, im Sinne von: „Genau, wir ackern mit Ochsen, weil wir noch keinen Traktor haben.“ Auch das zweite Beispiel in 0072 passt dazu, denn S2 bestätigt indirekt wiederum, was S1 bereits annimmt: „Genau, wir ziehen keine Schweine, weil das bei uns nicht geklappt hat.“ In Zeile 0168 antwortet S2 auf eine Frage. Hier wird ebenfalls die indirekte Annahme von S1, dass S2 einen oder mehrere der Begriffe für Pferd kenne, von S2 bestätigt. Vor diesem Hintergrund kommentiert S2 dann auch das Lexem Heiter: „Genau, wir sagen Gaul und wir kennen auch Heiter, aber der Ausdruck ist nicht so schön."

35. $0009 \quad \mathrm{~S} 1 \quad$ Ja, Ihr ackert mit Ochsen.

$0010 \quad$ S2 ja, kein Traktor haben wir noch nicht.

[...]

$0065 \quad$ S1 Haltet Ihr auch Schweine?

$0066 \quad \mathrm{~S} 2 \quad \mathrm{Ja}$, viele.

$0067 \quad \mathrm{~S} 1 \quad$ Viele, wie viele?

$0068 \quad$ S2 Ja, ziemlich haben wir jetzt.

\footnotetext{
10 Der ,some/any-Unterschied““ wurde erstmals von Bolinger (1960) erwähnt.

${ }^{11}$ Ich verdanke den Hinweis auf den positiven Erwartungshorizont Karin Donhauser.
} 


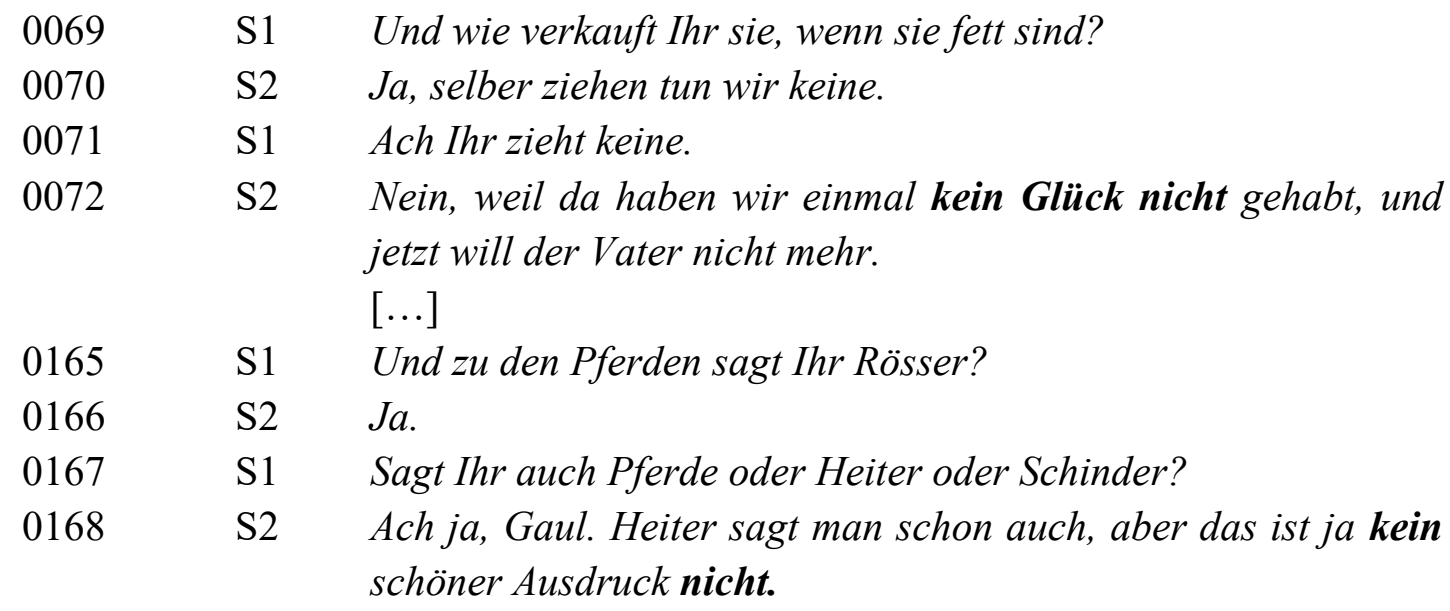

(Bairisch; Leutstetten Lkr. Starnberg; ZW: E_00902_S_01026)

Im zweiten Interview (36) finden wir ein Beispiel für eine $\mathrm{dN}$ mit und ohne $\mathrm{dN}$ vor. In 0034 wird keine dN geäußert, da S1 erstaunt ist, dass Heiligabend noch als Fastentag gilt und die Frage in 0033 vor diesem Hintergrund geäußert wird, i. e. S1 erwartet eine positive Antwort auf seine Frage; eine Antwort im Sinne von: „Nein, wenn es erlaubt war, wurde der Fastentag nicht gehalten." S2 bestätigt nicht S1s Vermutung; die Antwort wird nicht gegen einen positiven Erwartungshorizont geäußert, was sich auch in der Verwendung der Interjektion $\mathrm{Mei}$ äußert. Es ist nicht möglich zu formulieren: „Ja, alle Tage isst man kein Fleisch.“ Im Falle der $\mathrm{dN}$ in 0058 antwortet S2 auf den Erwartungshorizont von S1, wobei S2 schon mit dem vorangehenden Satz mit den Bohnenknödeln (und in 0056) S1 klargemacht hat, dass kein Fisch gegessen wird. I. e. die Antwort wird gegen einen positiven oder neutralen, sicher aber nicht gegen einen negativen Erwartungshorizont geäußert. Man könnte auch sagen: „Nein, keine Fische gibt es nicht.“

36. $0029 \quad \mathrm{~S} 1 \quad$ Und was ist so am Heiligen Abend, was eßt Ihr denn am Heiligen Abend?

$0030 \quad$ S2 $\quad J a$, da ist noch Fastentag, nicht.

$0031 \quad$ S1 Da ist noch Fastentag?

$0032 \quad \mathrm{~S} 2 \quad J a$.

$0033 \quad$ S1 Da ist noch Fastentag hier. Wird der immer noch gehalten, auch wo es erlaubt war, im Krieg zum Beispiel?

$0034 \quad$ S2 Ja, das hat es gar nicht anders gegeben. Mei, alle Tage isst man kein Fleisch. Da kann man ruhig diese Tage halten da, sagen wir hier, nicht.

[...]

$0055 \quad$ S1 Und in der Karwoche gibt es da besonderes Essen?

$0056 \quad$ S2 Nein, eigentlich nicht.

$0057 \quad$ S1 Am Karfreitag?

0058 S2 Gibt es halt meistens dann die Bohnenknödeln. Keine Fische gibt es nicht.

(Bairisch; Pfannstiel, Lkr. Rosenheim; ZW: E_00970_S_01094)

Schließlich noch ein drittes Beispiel: In Interview (37) wird die $\mathrm{dN}$ in einem monologischen Kontext geäußert; auch hier liegt jedoch ein positiver oder mindestens neutraler 
Erwartunghorizont vor, denn der Explorator ermutigt den Informanten zu Beginn, etwas über seine Heimat zu erzählen:
37. 0001
Herr NAME, Sie sind aus [(PAUSE)] in NAME geboren, und wohnen jetzt in NAME, können Sie irgendwas von Ihrer Heimat erzählen?
S2
$[\ldots]$
dem heiligen Ulrich geholfen und haben sie die heidnischen Ungarn vertrieben bis in unsere Gegend her und dann hat der Ulrich, weil's grad so heiß war, hat er geschaut, daß er seine Verwundeten und sein Vieh mit Wasser versorgen kann, dann ist aber leider kein Brunnen nicht dagewesen und das Wasser, wo da war, haben die $U$ Ungarn alles vergiftet, dann ist er auf die Knie hingefallen und $[\ldots]$

(Bairisch; Wildenroth, Lkr. Fürstenfeldbruck; ZW: E_00896_S_01020)

Diese Gedanken zur Funktion der dN deuten darauf hin, dass die Variation bei der dN mit Satznegation nicht frei ist, sondern dass die Pragmatik eine wichtige Rolle spielt. Eine syntaktische oder semantische Konditionierung scheint dagegen nicht vorzuliegen.

\section{$5 \quad$ Zusammenfassung}

In diesem Artikel wurde gezeigt, dass die dN bei Sprechern verschiedener deutscher Mundarten möglich ist. Zwischen den einzelnen Sprechern und auch innerhalb eines Sprechers liegt jedoch Variation vor: Während fast alle Sprecher bis auf jene des Alemannischen nur die dN mit Satznegation verwenden, ist bei den alemannischen Sprechern die präferierte Form die dN ohne Satznegation. Eine Untersuchung möglicher Kontexte für die dN bei den Sprechern des Bairischen und Alemannischen zeigt zudem, dass diese Kontexte (für die jeweils andere Negationsstrategie) von den Sprechern nicht genutzt werden: Obwohl die bairischen Sprecher mit Satznegation die dN ohne Satznegation nutzen könnten (mögliche Kontexte liegen vor), verwenden sie nur die dN mit Satznegation. Genau dasselbe gilt für die alemannischen Sprecher mit dN ohne Satznegation: Auch hier finden sich mögliche, nicht-realisierte Kontexte für die dN mit Satznegation. Des weiteren wird die dN mit Satznegation von den Sprechern des Bairischen nicht in jedem möglichen Kontext eingesetzt, sondern kann wahrscheinlich nur vor einem positiven Erwartungshorizont geäußert werden, da der Sprecher damit eine Bestätigung/Zustimmung ausdrücken möchte. Die dN mit Satznegation ist also pragmatisch konditioniert und nicht frei optional. Schließlich wird die dN mit Satznegation bei allen Sprechern fast ausschließlich mit dem n-Indefinitum koa ,kein' bzw. keen ,kein` etc. realisiert, bei der dN ohne Satznegation sind dagegen bei einem einzelnen Sprecher verschiedene Kombinationen an Indefinita möglich. Damit weisen diese Ergebnisse darauf hin, dass bei den Sprechern des Oberdeutschen (und wahrscheinlich auch in den anderen deutschen Mundarten) - entgegen bisheriger Annahmen aus der typologischen Forschung - keine Korrelation zwischen den beiden Typen vorliegt und die Verwendung des einen Typs nicht die des anderen Typs bedingt. 


\section{Literaturverzeichnis}

Abraham, Werner (2000): „Mehrfachnegation (MN) im Deutschen und Afrikaans“. In: Beckmann, Susanne/König, Peter-Paul/Wolf, Georg (eds.): Sprachspiel und Bedeutung. Festschrift für Franz Hundsnurscher zum 65. Geburtstag. Tübingen, Niemeyer: 221-226.

Bayer, Josef (1990): "What Bavarian negative concord reveals about the syntactic structure of German”. In: Mascaró, Joan/Nespor, Marina (eds.): Grammar in Progress. Dordrecht, Foris: $13-23$.

Bellmann, Günter (1964): „Wege und Möglichkeiten der Schallaufnahme ostdeutscher Mundarten heute. Zur Tonbandaufnahme der Vertriebenenmundarten“. Zeitschrift für Mundartforschung 31: 62-79.

Bernini, Giuliano/Ramat, Paolo (1996): Negative Sentences in the Languages of Europe. A Typological Approach. Berlin/New York: de Gruyter.

den Besten, Hans (1986): "Double negation and the genesis of Afrikaans". In: Muysken, Pieter/Smith, Norval (eds.): Substrata versus universals in creole genesis. Amsterdam, John Benjamins: 185-230.

Boliner, Dwight (1960): "Linguistic science and linguistic engineering." Word 16/3: 374-391. Brandenburg-Berlinisches Wörterbuch (1976-2001). Komm., begr. u. angelegt von Anneliese Bretschneider unter Einschluß d. Sammlungen von Hermann Teuchert. Fortgesetzt von Gerhard Ising. Bearb. unter d. Leitung von Joachim Wiese. Berlin: Akademie-Verlag.

Braunstein, Hermann (1978): Der Dialekt des Dorfes Schutterwald (Ortenaukreis). Grammatik und Wortschatz. Schutterwald: Selbstverlag.

Breitbarth, Anne (2013): "Negation in the history of Low German and Dutch". In: Willis, David/Lucas, Christopher/Breitbarth, Anne (eds.): The history of Negation in the Languages of Europe and the Mediterranean. Vol. 1: Case Studies. Oxford, Oxford University Press: 190238.

Bresnan, Joan/Deo, Ashwini/Sharma, Devyani (2007): "Typology in variation: a probabilistic approach to be and n't in the Survey of English Dialects". English Language and Linguistics $11 / 2,301-346$.

Diesing, Molly (1992): Indefinites. Cambridge, MA: MIT Press.

Gebhardt, August (1907): Grammatik der Nürnberger Mundart. Leipzig: Breitkopf \& Härtel.

Haspelmath, Martin (1997): Indefinite Pronouns. Oxford: Clarendon Press.

Heim, Irene (1982): The Semantics of Definite and Indefinite Noun Phrases. PhD Dissertation. University of Massachusetts at Amherst.

IDS Mannheim: Datenbank für Gesprochenes Deutsch (Hg.): DR-Korpus: Deutsche Mundarten DDR. https://dgd.ids-mannheim.de/dgd/ [03.07.2018]

IDS Mannheim: Datenbank für Gesprochenes Deutsch (Hg.): ZW-Korpus: Zwirner-Korpus. https://dgd.ids-mannheim.de/dgd/ [03.07.2018]

Jespersen, Otto (1917): Negation in English and other languages. Kœbenhavn: Hœst.

Kamp, Hans (1981): "A Theory of Truth and Semantic Representation". In: Groenendijk, Jeroen A.G./Janssen, Theo M.V./Stockhof, M.B.J. (eds.): Formal Methods in the Study of Language. Amsterdam, Mathematical Centre Tract 132: 277-322.

König, Werner/Schrambke, Renate (1999): Die Sprachatlanten des schwäbisch-alemannischen Raumes: Baden-Württemberg, Bayerisch-Schwaben, Elsaß, Liechtenstein, Schweiz, Vorarlberg. Bühl: Konkordia-Verlag. (= Themen des Landeskunde 8). 
Lakoff, Robin (1969): “Some reasons why there can't be any some-any rule". Language 45: 608-615.

Schmidt, Jürgen Erich/Herrgen, Joachim (2011): Sprachdynamik. Eine Einführung in die moderne Regionalsprachenforschung. Berlin: Erich Schmidt (= Grundlagen der Germanistik 49).

Staedele, Alfons (1927): Syntax der Mundart von Stahringen. Lahr i. Br.: Schauenburg.

Südwestdeutscher Sprachatlas (SSA): Interviews mit Sprechern aus ausgewählten Orten Südwestdeutschlands (Dialektabfrage, Interviews, auch Spontansprache). Datum der Erhebung: 1970er und 1980er Jahre. Forschungsstelle Sprachvariation in Baden-Württemberg, Universtät Freiburg.

Vogt, Friedrich E. (1977): Schwäbisch in Laut und Schrift. Eine ergründende und ergötzliche Sprachlehre. Stuttgart: Steinkopf.

Weber, Albert (1987): Zürichdeutsche Grammatik und Wegweiser zur guten Mundart. 3. Aufl. Zürich: Schweizer Spiegel Verlag.

Weiß, Helmut (1998): Syntax des Bairischen. Studien zur Grammatik einer natürlichen Sprache. Tübingen: Niemeyer.

van der Wouden, Ton/Zwarts, Frans (1993): "A Semantic Analysis of Negative Concord." In: Lahiri, Uptal/Wyner, Adam (eds.): SALT III. Ithaca, N.Y., Cornell University: 202-219. Wörterbuch der obersächsischen Mundarten (1994-2003). Begr. v. Theodor Frings u. Rudolf Große, unter der Leitung von Gunter Bergmann. Berlin: Akademie-Verlag.

Zehetner, Ludwig (1985): Das bairische Dialektbuch. München: Beck.

Zeijlstra, Hedde (2004): Sentential negation and negative concord. $\mathrm{PhD}$ dissertation, University of Amsterdam.

Zwirner, Eberhard/Bethge, Wolfgang (1958): Erläuterungen zu den Texten. Göttingen: Vandenhoeck \& Ruprecht. (=Lautbibliothek der deutschen Mundarten 1). 